\title{
Die Datenqualität lässt noch zu wünschen übrig
}

\author{
Intensiv tauschten sich kürzlich auf dem 3. DRG-Forum in Bern* 208 Fachleute aus \\ der Schweiz, Deutschland, Österreich und Grossbritannien über den aktuellen \\ Stand von DRG, Probleme und wie man sie lösen kann, aus.
}

\section{Felicitas Witte}

Freie Medizinjournalistin Fotos: Peter Brandenberger

* 3. DRG-Forum SchweizDeutschland, im Internet unter www.medicongress.ch/ kongresse/2014/Rueckblicke/ DRG_Forum_3.php
Korrespondenz:

Dr. med. Felicitas Witte Seefeldstrasse 285 CH-8008 Zürich

felicitas.witte[at]web.de

\section{Bessere Datenqualität vonnöten}

«Die meisten Akteure haben noch nicht verstanden, dass wir von einem Kostenerstattungs- zu einem Preisprinzip wechseln», sagte Willy Oggier, Gesundheitsökonom aus Küsnacht und Mit-Initiator des DRG-Forums. Viele Punkte seien immer noch nicht geklärt oder nicht gut umgesetzt, kritisierte Oggier. Die gelieferten Daten zur Ermittlung der Fallschwere seien beispielsweise noch von zu schlechter Qualität, und Extremkosten würden nicht gut abgebildet. «Ausserdem hat die Schweiz durch die Trennung in Grund- und Zusatzversicherungen einen Nachteil gegenüber Deutschland», sagte Oggier. "Kaum ein Versicherer hat ein Interesse an zu guter Qualität in der Grundversicherung, weil dies damit als VerkaufsArgument für die finanziell attraktiveren Zusatzversicherungen wegfallen würde.»

Als 2012 die einheitliche Tarifstruktur SwissDRG eingeführt wurde, so Oggier, wurde bei der Anwendung schnell klar, dass im Vergleich zur deutschen Tarifstruktur einige Mängel bestehen. Das beträfe insbesondere die Ungenauigkeit der Tarifstruktur. «Die neuen Regeln benachteiligen Spitäler, die hochspezialisierte Leistungen anbieten», erklärte Oggier. «Hochspezialisierte Behandlungen werden selten durchgeführt und sind im System nicht richtig abgebildet. Wir bräuchten mehr Diagnosegruppen und mehr Zusatzentgelte, wenn wir eine gute Tarifstruktur für Spitalvergleiche haben wollen.» Zudem sei nicht klar, wie im neuen System medizinische Innovationen finanziert werden sollen. Neue Anreize müssten geschaffen werden, forderte Oggier, um SwissDRG zu verbessern. So sei beispielsweise die Frage zu stellen, ob die höheren Base Rates für Universitätsspitäler $\mathrm{zu}$ hoch seien und miterklärten, warum man in der Schweiz so ungenügende Datengrundlagen für die Berechnung von Zusatzentgelten habe. "Nur mit mehr Transparenz hat SwissDRG Erfolg, dazu ist eine sorgfältige Datenlieferung essentiell.» In Frage stellte Oggier auch die Rolle des Preisüberwachers bei einvernehmlich zustande gekommenen Tarifen. Dort gebe es heute nämlich sowohl auf der Krankenversicherer- als auch auf der Spitalseite mehr Wettbewerb.

Bessere Datenqualität mahnte auch Constanze Hergeth an, die Leiterin des Geschäftsbereiches Akutsomatik bei der SwissDRG AG in Bern. 2012 war das erste Jahr, in dem in der gesamten Schweiz nach DRG abgerechnet wurde. «Wir hatten gehofft, dass es zu einem grossen Sprung in der Datenqualität

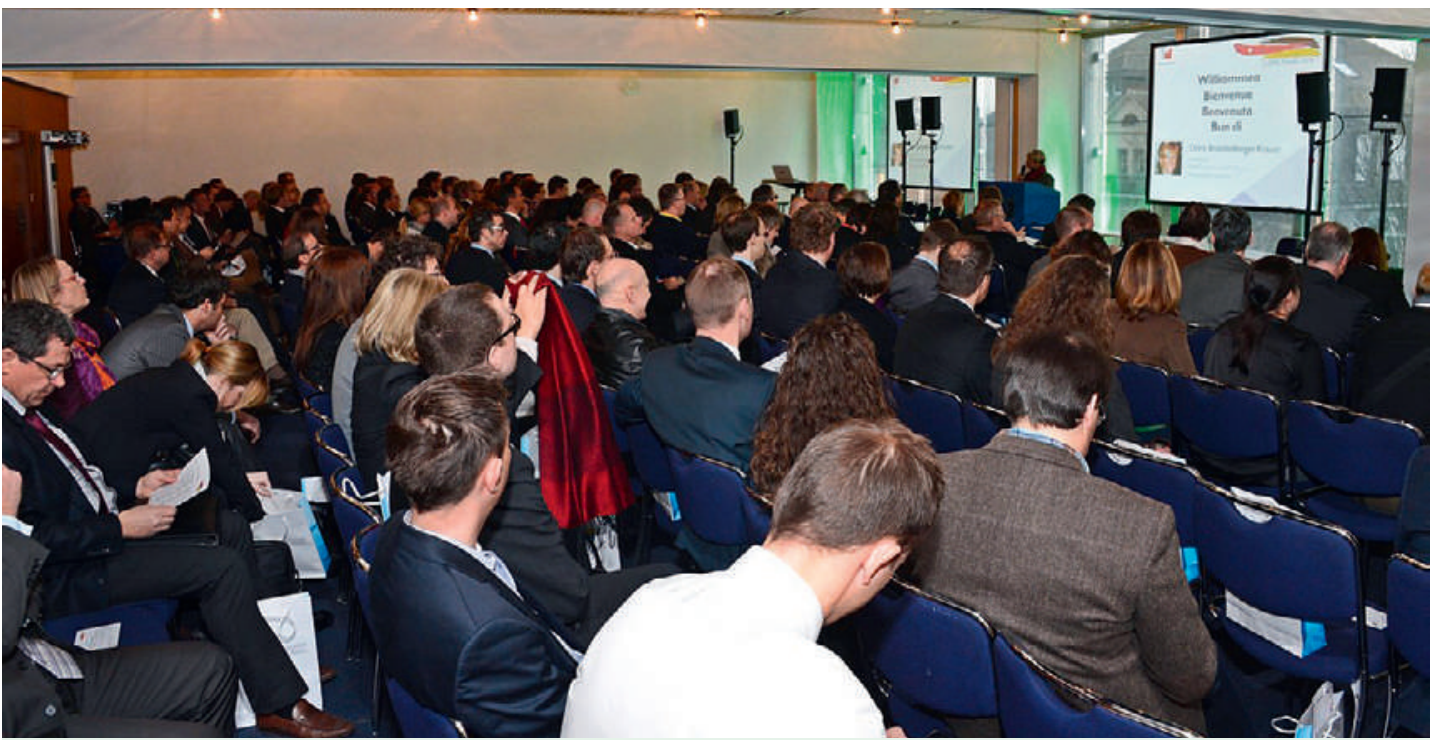

Experten aus Deutschland, Österreich und der Schweiz schilderten ihre Erfahrungen mit DRGs. 


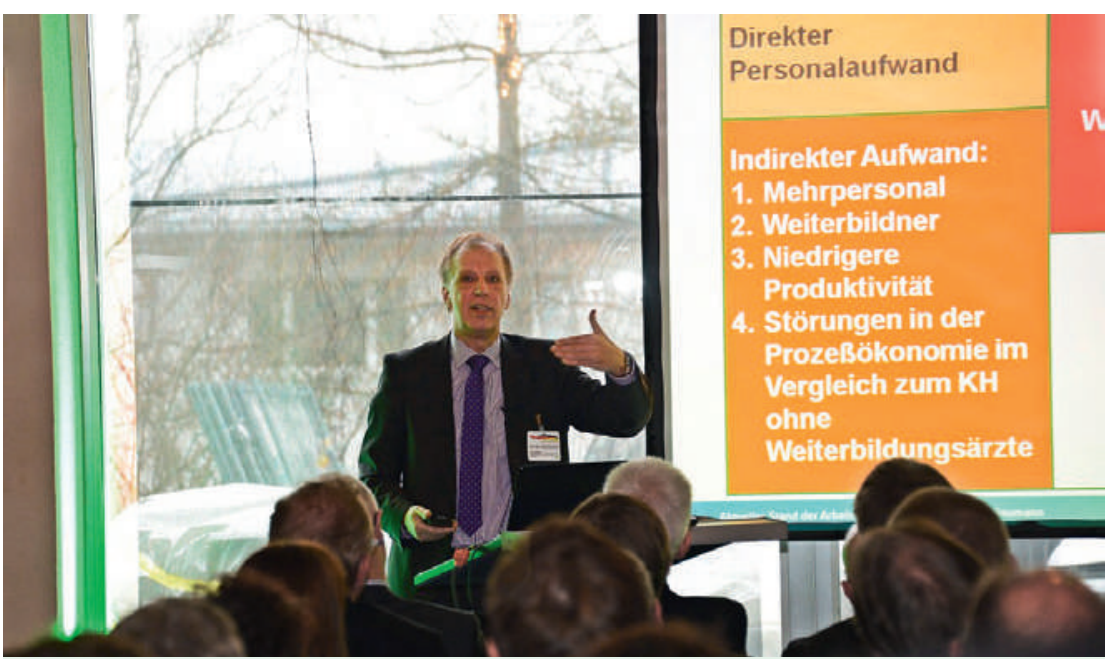

Holger Baumann, Vize-Präsident der Medizinischen Hochschule Hannover und designierter Geschäftsleitungs-Vorsitzender des Inselspitals Bern, kennt die Arbeit mit DRGs seit 2003.

kommen würde», sagte Hergeth. «Aber vielleicht war es nur ein Hüpfer. Wir sind bei der Datenqualität noch nicht dort, wo wir hinwollen.» 103 Spitäler haben die Daten des Jahres 2012 an SwissDRG be- durchgeführt wurde!» Dass 18,9 Prozent der Prozedurenkodes mit .99 klassifiziert worden seien, also mit «sonstige», könne sie etwas verstehen. Bei einigen Prozeduren sei es leider so, dass sie noch nicht gut in der CHOP-Klassifikation abgebildet seien. Aber die SwissDRG AG habe im vergangenen Jahr ihre Hausaufgaben gemacht. «Wir haben vieles umgebaut und verbessert.» So sind jetzt aufwendige nichtoperative Behandlungen besser abgebildet: Aufgewertet wurden zum Beispiel Fälle mit einer «Neurologischen Komplexbehandlung des akuten Schlaganfalles ausserhalb einer spezialisierten Einheit» sowie komplexe Pneumonie- und Beatmungsfälle.

\section{Erfahrungen aus Deuschland}

Deutschland hat der Schweiz im Bereich DRG einiges voraus - schliesslich gibt es hier das DRG-System schon seit 2003. Einer, der sich damit am besten auskennt, ist Holger Baumann, Vize-Präsident der Medizinischen Hochschule Hannover (MHH) und designierter Geschäftsleitungs-Vorsitzender des Inselspitals Bern. «Wir waren eine der ersten Unikliniken in Deutschland, die sich 2004 an der Kalkulation beteiligt haben», erzählt Baumann. «Wir haben uns als Motor der Unikliniken gesehen.» Hohe Erwartungen

\section{«Unispitäler brauchen viel mehr Personal für die Ausbildung - aber finanziell abgedeckt wird das nicht.» (Holger Baumann)}

richtet, das entspricht etwa 80,7\% aller Schweizer stationären Fälle in der Akutsomatik.

Die Spitäler würden häufig aber nur ungenaue oder unvollständige Informationen liefern, kritisierte Hergeth. Zum Beispiel dokumentierten zu wenige Spitäler Komplexbehandlungen wegen multiresistenter Erreger. «Glaube ich den Zahlen, scheint es in der Schweiz die paradiesische Situation zu geben, dass wir keine Infektionen mit multiresistenten Erregern haben», sagte Hergeth. «In Wirklichkeit werden sie aber wohl nicht ausreichend erfasst.»

Leider gebe es zudem oft noch Probleme in der Kostenerfassung: Die kodierten Leistungen eines Spitals passen nicht zu den erfassten Kosten.

«Der Patient erscheint aufgrund der Kodierung nahezu gesund, und auf der Kostenerfassung sehe ich dann Arzt- und Pflegekosten von 6000 bis 10000 Franken pro Tag. Da stimmt etwas nicht.» Die Spitäler müssten solche Inkonsistenzen bereits prüfen, bevor sie die Daten an die SwissDRG AG weitergäben, forderte Hergeth. Häufig werden zudem ungültige Kodes oder unspezifische Kodes verwendet. So wurden 6,44 Prozent aller Prozedurenkodes mit «.00», klassifiziert, also «nicht näher bezeichnet». «Das macht mich sprachlos - es kann doch nicht sein, dass das Spital häufig nicht weiss, was für eine Behandlung habe die Politik damals gehabt, aber man dürfe nicht $\mathrm{zu}$ viel erwarten. Aus Sicht eines Unispitals sieht Baumann hauptsächlich vier Probleme der Finanzierung: Erstens seien die Extremkostenfälle nicht richtig abgebildet, die hauptsächlich für die Unterfinanzierung vieler Unispitäler verantwortlich seien. Zweitens würden die Spitäler für die Notfallversorgung nicht kostendeckend vergütet. Drittens sei immer noch unklar, wer die ärztliche Weiterbildung finanziert. «Unispitäler brauchen viel mehr Personal für die Ausbildung - aber finanziell abgedeckt wird das nicht.» Viertens würden Innovationen nicht oder nicht ausreichend vergütet. «Neue Techniken, die vermehrt eingesetzt werden, um todkranke Menschen am Leben zu erhalten, werden nicht finanziert», sagte Baumann. So gebe es zum Beispiel ein neues Perfusionsset für die Lungentransplantation, das das entnommene Organ so frisch wie möglich hält, bevor es eingepflanzt wird. Das Einzelsystem kostet 41000 Euro, eine Gegenfinanzierung erhielt die MHH nicht. Das deutsche DRG-Institut InEK forderte zunächst eine Klärung auf Bundesebene. In der MHH führte das 2013 zu einer Finanzierungslücke von 416000 Euro. Das System DRG sei ein fliessender Prozess, den es ständig zu verbessern gelte, sagte Baumann. Das gilt nicht nur qualitativ, sondern 
auch quantitativ: Denn demnächst soll es leistungsabhängige Tarife auch in der Psychiatrie und in der Rehabilitation geben.

\section{Kostengruppen für die Psychiatrie}

«Schon lange war man sich bewusst, dass psychiatrische Leiden keine genügende Vorhersagequalität zur Behandlungsdauer erlauben», berichtete Jürg UngerKöppel, Mitglied der Geschäftsleitung der Psychiatrischen Dienste Aargau AG. «So gab es keine Grundlage für die Festlegung von DRGs.» 2006 griff die Aktivkonferenz Psychiatrie $\mathrm{H}+$ das Thema auf und versuchte 2008 ein erstes Entgeltungssysystem Psysuisse zu entwickeln. Das ergab aber keine genügend differenzierenden Werte, und 2011 bekamen GD Zürich und $\mathrm{H}+$ zusammen den Auftrag, einen neuen, leistungsbezogenen Tarif für die Psychiatrie zu entwickeln - das Projekt TARPSY. Die Entwickler bemühten sich um möglichst homogene Kostengruppen in

\section{«Tageskliniken müssten zwingend zum TARPSY dazugehören.» \\ (Jürg Unger-Köppel)}

Form von Diagnosegruppen, Psychiatric Cost Groups oder PCGs. Der Schweregrad wird unterteilt in Regelund Intensivbehandlungen. «Das Problem ist, dass gleiche psychiatrische Krankheiten unterschiedliche Verläufe haben», sagte Unger-Köppel. «Ausserdem sind die Behandlungsalgorithmen wenig standardisiert.» 2012 und 2013 wurde für TARPSY in mehreren Erwachsenen-psychiatrischen und zwei Kinderpsychiatrischen Kliniken Daten gesammelt. 20 PCGs konnten gebildet werden, das Modell sei grundsätzlich valide, so Unger-Köppel. 2014 soll das System weiterentwickelt und 2018 gestartet werden. Das TARPSY-System ist konform mit dem Krankenversicherungsgesetz (KVG), aber der Psychiater sieht zwei grundsätzliche Probleme: «Die heutige Finanzierungsstruktur ergibt aufgrund des Krankenversicherungsgesetzes falsche finanzielle Anreize in der Psychiatrie. Stationär vor ambulant ist für die Leistungserbringer günstiger, und ambulant vor stationär ist für die Patienten günstiger.» Gemäss KVG gelte auch die Tagesklinische Behandlung als ambulant. «Das ist hochdefizitär und damit finanziell un- tragbar. Tageskliniken müssten zwingend zum TARPSY dazugehören», forderte Unger-Köppel im Namen der FMH. Als ganz grosse Vision wünscht er sich eine Integrierte Finanzierung: Eine Institution erhält einen Betrag pro Zeiteinheit für die integrierte Behandlung des Patienten und die Institution behandelt ihn nach den Anforderungen seiner Krankheit ambulant, stationär oder teilstationär. Im Ausland, etwa im deutschen Bundesland Niedersachsen, gäbe es Modellversuche, berichtete Unger-Köppel. «Bei uns müsste dazu aber das KVG angepasst werden oder Pilotprojekte müssten gestartet werden.»

\section{Ein neues Tarifsystem für Rehabilitation}

Das Tarifsystem in der Rehabilitation heisst ST Reha. Auch hier wird in zwei Schritten gruppiert, erst die Art der Behandlung, dann der Schweregrad. 14 Kliniken erhoben 2013 Daten, zurzeit sollen die Schweregrad-Klassifizierungen weiterentwickelt und Ende 2016 die Version 1.0 fertig sein. «Auch die Rehabilitation ist sehr heterogen», sagte Hansulrich Tschanz, Chefarzt Kardiologie am Berner Reha Zentrum Heiligenschwendi und Vertreter der FMH im Steuerungsausschuss ST Reha. «Es gibt keine einheitliche Nomenklatur und wenig breit anerkannte Normen.» Ausserdem sei Rehabilitation ständig in Bewegung. «Die Paraplegiologie verstand sich lange als Teil der neurologischen Rehabilitation. Seit kurzem entwickelt sie sich aber zunehmend eigenständig. Und auch die onkologische und die internistische Rehabilitation definieren sich neu.» Die Funktionalität, die für die Tarifermittlung wichtig ist, sei aufwendig zu messen für Personal und Patienten und könne zulasten der Therapie gehen. «Die Patienten sind wenig begeistert.»

Die hier geschilderten Themen geben nur einen kleinen Einblick in die Vielfalt des Kongresses. Experten aus Deutschland und Österreich schilderten ihre Erfahrungen mit DRG, in den Pausen und am Abendessen diskutierte man intensiv. «Speziell an dem DRG-Forum ist die offene Diskussion zwischen Teilnehmern und Referenten», sagt Doris Brandenberger von MediCongress $\mathrm{GmbH}$, die gemeinsam mit Willy Oggier das DRG-Forum ins Leben rief. «Der Kongress hat einmal mehr aufgezeigt, dass wir vieles aus Deutschland lernen können und es sich lohnt, über die Grenze zu schauen. Ein Miteinander anstelle eines Gegeneinander lohnt sich immer!»

Das 4. DRG-Forum Schweiz-Deutschland findet am 29. und 30.1.2015 in Bern statt. 